\title{
OPERATORS WITH WENTZELL BOUNDARY CONDITIONS AND THE DIRICHLET-TO-NEUMANN OPERATOR
}

\author{
TIM BINZ AND KLAUS-JOCHEN ENGEL
}

\begin{abstract}
In this paper we relate the generator property of an operator $A$ with (abstract) generalized Wentzell boundary conditions on a Banach space $X$ and its associated (abstract) Dirichlet-to-Neumann operator $N$ acting on a "boundary" space $\partial X$. Our approach is based on similarity transformations and perturbation arguments and allows to split $A$ into an operator $A_{00}$ with Dirichlet-type boundary conditions on a space $X_{0}$ of states having "zero trace" and the operator $N$. If $A_{00}$ generates an analytic semigroup, we obtain under a weak Hille-Yosida type condition that $A$ generates an analytic semigroup on $X$ if and only if $N$ does so on $\partial X$. Here we assume that the (abstract) "trace" operator $L: X \rightarrow \partial X$ is bounded what is typically satisfied if $X$ is a space of continuous functions. Concrete applications are made to various second order differential operators.
\end{abstract}

\section{INTRODUCTION}

The generation of analytic semigroups by differential operators with generalized Wentzell boundary conditions on spaces of continuous functions attracted the interest of many authors, and we refer, e.g., to [CM98], [FGGR02], [Eng03], [EF05], [FGG ${ }^{+}$10]. For their derivation and physical interpretation we refer to [Gol06]. The present paper is a continuation and improvement of [EF05] where we introduced a general abstract framework to deal with this problem. Before recalling this setting we consider the following typical example in order to explain the basic ideas and the goal of our approach.

Take a smooth bounded domain $\Omega \subset \mathbb{R}^{n}$. Then consider on $\mathrm{C}(\bar{\Omega})$ the Laplacian $\Delta_{m}$ with "maximal" domain $D\left(\Delta_{m}\right):=\left\{f \in \mathrm{C}(\bar{\Omega}): \Delta_{m} f \in \mathrm{C}(\bar{\Omega})\right\}$, where the derivatives are taken in the distributional sense. Finally, let $\frac{\partial}{\partial n}: D\left(\frac{\partial}{\partial n}\right) \subset \mathrm{C}(\bar{\Omega}) \rightarrow \mathrm{C}(\partial \Omega)$ be the outer normal derivative, $\beta<0$ and $\gamma \in \mathrm{C}(\partial \Omega)$. In this setting we define the Laplacian $A \subset \Delta_{m}$ with generalized Wentzell boundary conditions by requiring

$$
f \in D(A) \quad:\left.\Longleftrightarrow \Delta_{m} f\right|_{\partial \Omega}=\beta \cdot \frac{\partial}{\partial n} f+\left.\gamma \cdot f\right|_{\partial \Omega} .
$$

Our approach decomposes a function $f \in \mathrm{C}(\bar{\Omega})$ into the (unique) sum $f=f_{0}+h$ of a function $f_{0}$ vanishing at the boundary $\partial \Omega$ and a harmonic function $h$ having the same trace as $f$. In other words, if $L: \mathrm{C}(\bar{\Omega}) \rightarrow \mathrm{C}(\partial \Omega), L f:=\left.f\right|_{\partial \Omega}$ denotes the trace operator, then $f_{0} \in \operatorname{ker} L=\mathrm{C}_{0}(\Omega)$ while $h \in \operatorname{ker}\left(\Delta_{m}\right)$. Since $h$ is uniquely determined by its trace, it can be identified with its boundary value $x:=L h$. Hence, every $f \in \mathrm{C}(\bar{\Omega})$ corresponds to a unique pair $\left(\begin{array}{l}f_{0} \\ x\end{array}\right) \in \mathrm{C}_{0}(\Omega) \times \mathrm{C}(\partial \Omega)$.

To formalize this decomposition we introduce an abstract "Dirichlet operator" $L_{0}: \mathrm{C}(\partial \Omega) \rightarrow$ $\mathrm{C}(\bar{\Omega})$. To this end we consider for a given "boundary function" $x \in \mathrm{C}(\partial \Omega)$ the Dirichlet problem

$$
\left\{\begin{array}{l}
\Delta_{m} f=0, \\
\left.f\right|_{\partial \Omega}=x .
\end{array}\right.
$$

Date: November 28, 2018.

1991 Mathematics Subject Classification. 47D06, 34G10, 47E05, 47F05.

Key words and phrases. Wentzell boundary conditions, Dirichlet-to-Neumann operator, analytic semigroup. 
This system admits a unique solution $f \in \mathrm{C}(\bar{\Omega})$, so by setting $L_{0} x:=f$ we obtain an operator $L_{0} \in \mathcal{L}(\mathrm{C}(\partial \Omega), \mathrm{C}(\bar{\Omega}))$. For $f \in \mathrm{C}(\bar{\Omega})$ we then have $f=f_{0}+h$ where $f_{0}:=\left(\operatorname{Id}-L_{0} L\right) f$ and $h=L_{0} x$ for $x:=L f$. By (1.1) it then follows (for the details see Step 1 below in the proof of Theorem 3.1) that $A$ on $\mathrm{C}(\bar{\Omega})$ transforms into an operator matrix $\mathcal{A}$ on $\mathrm{C}_{0}(\Omega) \times \mathrm{C}(\partial \Omega)$ of the form

$$
\mathcal{A}:=\left(\begin{array}{cc}
\Delta_{m} & 0 \\
0 & N
\end{array}\right)+\mathcal{P}
$$

with some appropriate "non-diagonal" domain $D(\mathcal{A}) \subset \mathrm{C}_{0}(\Omega) \times \mathrm{C}(\partial \Omega)$, see [Eng98], [Eng99], [Nag90]. Here $\mathcal{P}$ denotes an unbounded perturbation while $N:=\beta \cdot \frac{\partial}{\partial n} \cdot L_{0}$ is the so called Dirichlet-to-Neumann operator on $\mathrm{C}(\partial \Omega)$, see [Esc94], [Tay96, Sect. 12.C]. That is, $N x$ is obtained by applying the Neumann boundary operator to the solution $f$ of the Dirichlet problem (1.2).

Using perturbation arguments one can show that $\mathcal{A}$, hence also $A$, generate analytic semigroups if and only if the Dirichlet Laplacian $\Delta_{00}$ on $\mathrm{C}_{0}(\Omega)$ and the Dirichlet-to-Neumann operator $N$ on $\mathrm{C}(\partial \Omega)$ do so. This means that we decoupled the operator $A \subset \Delta_{m}$ with generalized Wentzell boundary conditions on $X:=\mathrm{C}(\bar{\Omega})$ into an operator $A_{00}:=\Delta_{00}$ with Dirichlet boundary conditions on $X_{0}:=\mathrm{C}_{0}(\Omega)$ and the Dirichlet-to-Neumann operator $N:=\beta \cdot \frac{\partial}{\partial n} \cdot L_{0}$ on the boundary space $\partial X:=\mathrm{C}(\partial \Omega)$.

Since it is well-known that $\Delta_{00}$ generates an analytic semigroup, our main result applied to this example yields that $A$ generates an analytic semigroup on $\mathrm{C}(\bar{\Omega})$ if and only if $N$ generates an analytic semigroup on $\mathrm{C}(\partial \Omega)$. Since the latter is true, see [Eng03, Sect. 2], we conclude that $A \subset \Delta_{m}$ with generalized Wentzell boundary condition (1.1) is the generator of an analytic semigroup. We mention that our approach also keeps track of the angle of analyticity and, in the above example, gives the optimal angle $\frac{\pi}{2}$.

This paper is organized as follows. In Section 2 we introduce our abstract setting and then state in Section 3 our main abstract generation result, Theorem 3.1. In the following Section 4 we show that the generator property of operators with generalized Wentzell boundary conditions is invariant under "small" perturbations with respect to the action as well as the domain, cf. Theorem 4.2 and Theorem 4.3. For these proofs we study in Lemma 4.6 and Proposition 4.7 how the Dirichlet- and Dirichlet-to-Neumann operator, respectively, behaves under relatively bounded perturbations. Finally, in Section 5 we apply our abstract results to second order differential operators on $\mathrm{C}\left([0,1], \mathbb{C}^{n}\right)$, the Banach space-valued second-order derivative, a perturbed Laplacian with generalized Wentzell boundary conditions and uniformly elliptic operators on $\mathrm{C}(\bar{\Omega})$. Our notation follows the monograph [EN00].

\section{The Abstract Setting}

As in [EF05, Section 2], the starting point of our investigation is the following

\section{Abstract Setting 2.1. Consider}

(i) two Banach spaces $X$ and $\partial X$, called state and boundary space, respectively;

(ii) a densely defined maximal operator $A_{m}: D\left(A_{m}\right) \subset X \rightarrow X$;

(iii) a boundary (or trace) operator $L \in \mathcal{L}(X, \partial X)$;

(iv) a feedback operator $B: D(B) \subseteq X \rightarrow \partial X$.

Using these spaces and operators we define the operator $A^{B}: D\left(A^{B}\right) \subset X \rightarrow X$ with abstract generalized Wentzell boundary conditions by

$$
A^{B} \subseteq A_{m}, \quad D\left(A^{B}\right):=\left\{f \in D\left(A_{m}\right) \cap D(B): L A_{m} f=B f\right\} .
$$

If $B=0$ the boundary conditions defined by (2.1) are called pure Wentzell boundary conditions. For an interpretation of Wentzell- as "dynamic boundary conditions" we refer to [EF05, Sect. 2]. 
To fit the example from the introduction into this setting it suffices to choose $X:=\mathrm{C}(\bar{\Omega})$, $\partial X:=\mathrm{C}(\partial \Omega), A_{m}:=\Delta_{m}, L f:=\left.f\right|_{\partial \Omega}$ and $B:=\beta \cdot \frac{\partial}{\partial n}+\gamma \cdot L$.

In the sequel we need the (in general non-densely defined) operator $A_{0}: D\left(A_{0}\right) \subset X \rightarrow X$ defined by

$$
A_{0} \subseteq A_{m}, \quad D\left(A_{0}\right):=D\left(A_{m}\right) \cap \operatorname{ker}(L) .
$$

In the example from the introduction $A_{0}$ is the Dirichlet Laplacian $\Delta_{0}$ on $\mathrm{C}(\bar{\Omega})$ with non-dense domain $D\left(A_{0}\right)=D\left(\Delta_{m}\right) \cap \mathrm{C}_{0}(\Omega)$.

\section{Assumptions 2.2.}

(i) The operator $A_{0}$ is a weak Hille-Yosida operator on $X$, i.e. there exist $\lambda_{0} \in \mathbb{R}$ and $M>0$ such that $\left[\lambda_{0}, \infty\right) \subset \rho\left(A_{0}\right)$ and

$$
\left\|\lambda R\left(\lambda, A_{0}\right)\right\| \leq M \quad \text { for all } \lambda \geq \lambda_{0} ;
$$

(ii) the operator $B$ is relatively $A_{0}$-bounded with bound 0, i.e., $D\left(A_{0}\right) \subseteq D(B)$ and for every $\varepsilon>0$ there exists $M_{\varepsilon}>0$ such that

$$
\|B f\|_{\partial X} \leq \varepsilon \cdot\left\|A_{0} f\right\|_{X}+M_{\varepsilon} \cdot\|f\|_{X} \quad \text { for all } f \in D\left(A_{0}\right) ;
$$

(iii) the abstract Dirichlet operator $L_{0}:=\left(\left.L\right|_{\operatorname{ker}\left(A_{m}\right)}\right)^{-1}: \partial X \rightarrow \operatorname{ker}\left(A_{m}\right) \subseteq X$ exists and is bounded, i.e., for every $x \in \partial X$ the abstract Dirichlet problem

$$
\left\{\begin{array}{c}
A_{m} f=0, \\
L f=x
\end{array}\right.
$$

admits a unique solution $f \in D\left(A_{m}\right)$ and $L_{0} x:=f$ defines an operator $L_{0} \in \mathcal{L}(\partial X, X)$.

We note that by [Gre87, Lem. 1.2] assumption (iii) is always satisfied if $A_{m}$ is closed, $L$ : $X \rightarrow \partial X$ is surjective and $A_{0}$ is invertible. Moreover, $L_{0} L \in \mathcal{L}(X)$ is a projection onto the subspace $\operatorname{ker}\left(A_{m}\right)$ along $X_{0}:=\operatorname{ker}(L)$ which induces the decompositions

$$
X=X_{0} \oplus \operatorname{ker}\left(A_{m}\right) \quad \text { and } \quad D\left(A_{m}\right)=D\left(A_{0}\right) \oplus \operatorname{ker}\left(A_{m}\right) .
$$

In the sequel we will need the following operators.

Notation 2.3. Define $G_{m}: D\left(G_{m}\right) \subset X \rightarrow X$ by

$$
G_{m} f:=A_{m} f-L_{0} B \cdot\left(\operatorname{Id}-L_{0} L\right) f, \quad D\left(G_{m}\right):=D\left(A_{m}\right) .
$$

Then for $* \in\{1,0,00\}$ we consider the restrictions $A_{*} \subset A_{m}$ and $G_{*} \subset G_{m}$ given by

$$
\begin{array}{ll}
A_{0}: D\left(A_{0}\right) \subset X \rightarrow X, & D\left(A_{0}\right):=\left\{f \in D\left(A_{m}\right): L f=0\right\}, \\
A_{1}: D\left(A_{1}\right) \subset X \rightarrow X, & D\left(A_{1}\right):=\left\{f \in D\left(A_{m}\right): L A_{m} f=0\right\}, \\
A_{00}: D\left(A_{00}\right) \subset X_{0} \rightarrow X_{0}, & D\left(A_{00}\right):=\left\{f \in D\left(A_{m}\right): L f=0, L A_{m} f=0\right\}
\end{array}
$$

and

$$
\begin{array}{ll}
G_{0}: D\left(G_{0}\right) \subset X \rightarrow X, & D\left(G_{0}\right):=D\left(A_{0}\right), \\
G_{1}: D\left(G_{1}\right) \subset X \rightarrow X, & D\left(G_{1}\right):=\left\{f \in D\left(G_{m}\right): L G_{m} f=0\right\}, \\
G_{00}: D\left(G_{00}\right) \subset X_{0} \rightarrow X_{0}, & D\left(G_{00}\right):=\left\{f \in D\left(G_{m}\right): L f=0, L G_{m} f=0\right\} .
\end{array}
$$

Observe that $G_{00} \subset G_{0}=A_{0}-L_{0} B$. In other words, $D_{*}$ for $D \in\{A, G\}$ and $* \in\{0,1,00\}$ is a restriction of $D_{m}$. For $*=0$ this restriction corresponds to abstract Dirichlet boundary conditions and for $*=1$ to pure Wentzell boundary conditions on $X$, while $D_{00}$ is the part of $D_{0}$ as well as of $D_{1}$ in $X_{0}$.

Finally, we define the abstract Dirichlet-to-Neumann operator $N: D(N) \subset \partial X \rightarrow \partial X$ by

$$
N x:=B L_{0} x, \quad D(N):=\left\{x \in \partial X: L_{0} x \in D(B)\right\} .
$$

This operator plays a crucial role in our approach. 


\section{The Main Result}

The following is our main abstract result. In contrast to [EF05, Thm. 3.1] it proves (besides further generalizations) that (a) $\Longleftrightarrow$ (b) and not only that (b) $\Rightarrow$ (a) in case $D=A$.

Theorem 3.1. Let $D \in\{A, G\}$. Then the following statements are equivalent

(a) $A^{B}$ given by (2.1) generates an analytic semigroup of angle $\alpha>0$ on $X$.

(b) $D_{0}$ is sectorial of angle $\alpha>0$ on $X$ and the Dirichlet-to-Neumann operator $N$ generates an analytic semigroup of angle $\alpha>0$ on $\partial X$.

(c) $D_{1}$ and $N$ generate analytic semigroups of angle $\alpha>0$ on $X$ and $\partial X$, respectively.

(d) $D_{00}$ and $N$ generate analytic semigroups of angle $\alpha>0$ on $X_{0}$ and $\partial X$, respectively.

Proof. By [EF05, Thm. 3.1] we have that (b) $\Rightarrow$ (a) for $D_{0}=A_{0}$. Since $A_{0}$ and $G_{0}$ only differ by a relatively bounded perturbation of bound 0, [EN00, Lem. III.2.6] implies that assumption (b) is equivalent for $D=A$ and $D=G$. This shows that (b) $\Rightarrow$ (a). The equivalences (b) $\Longleftrightarrow(\mathrm{c}) \Longleftrightarrow(\mathrm{d})$ for $D=A$ follow by [EF05, Lem. 3.3]. Now assume that $D=G$. Then by [EN00, Lem. III.2.5] there exists $\lambda \in \rho\left(G_{0}\right)$. Since $L$ is surjective, [Gre87, Lem. 1.2] implies that the Dirichlet operator for $G_{m}-\lambda$ exists. As before, [EF05, Lem. 3.3] now applied to $G_{0}-\lambda, G_{1}-\lambda$ and $G_{00}-\lambda$ gives the equivalence of (b), (c) and (d) for $D=G$. To complete the proof it suffices to verify that (a) $\Rightarrow$ (d) for $D_{00}=G_{00}$. We proceed in several steps where we put $X_{0}:=X_{0} \times \partial X$.

Step 1. The operator $A^{B}: D\left(A^{B}\right) \subset X \rightarrow X$ is similar to $\mathcal{A}: D(\mathcal{A}) \subset X_{0} \rightarrow X_{0}$ given by

$$
\mathcal{A}:=\left(\begin{array}{cc}
G_{0} & -L_{0} N \\
B & N
\end{array}\right), \quad D(\mathcal{A}):=\left\{\left(\begin{array}{l}
f \\
x
\end{array}\right) \in D\left(A_{0}\right) \times D(N): G_{0} f-L_{0} N x \in X_{0}\right\} .
$$

Proof. The operator

$$
T: X \rightarrow X_{0}, \quad \text { Tf }:=\left(\begin{array}{c}
f-L_{0} L f \\
L f
\end{array}\right)
$$

is bounded and invertible with bounded inverse

$$
T^{-1}: X_{0} \rightarrow X, \quad T^{-1}\left(\begin{array}{l}
f \\
x
\end{array}\right)=f+L_{0} x .
$$

We show that $\mathcal{A}=T A T^{-1}$. Using that $L L_{0}=\operatorname{Id}_{\partial X}, X_{0}=\operatorname{ker}(L)$ and $A_{m} L_{0}=0$ we have

$$
\begin{aligned}
\left(\begin{array}{l}
f \\
x
\end{array}\right) \in D(\mathcal{A}) & \Longleftrightarrow f \in D\left(A_{0}\right), x \in D(N) \text { and } A_{m} f-L_{0} B f-L_{0} N x \in X_{0} \\
& \Longleftrightarrow f \in D\left(A_{0}\right), x \in D(N) \text { and } L A_{m} f-B f-N x=0 \\
& \Longleftrightarrow f \in D\left(A_{0}\right), x \in D(N) \text { and } L A_{m}\left(f+L_{0} x\right)=B\left(f+L_{0} x\right) \\
& \Longleftrightarrow T^{-1}\left(\begin{array}{l}
f \\
x
\end{array}\right) \in D(A) \Longleftrightarrow\left(\begin{array}{l}
f \\
x
\end{array}\right) \in T D(A) .
\end{aligned}
$$

Moreover, for $\left(\begin{array}{l}f \\ x\end{array}\right) \in T D(A)=D(\mathcal{A})$ we obtain using that $f+L_{0} x \in D(A)$

$$
\begin{aligned}
T A T^{-1}\left(\begin{array}{l}
f \\
x
\end{array}\right) & =T A_{m}\left(f+L_{0} x\right) \\
& =\left(\begin{array}{c}
A_{m}\left(f+L_{0} x\right)-L_{0} L A_{m}\left(f+L_{0} x\right) \\
L A_{m}\left(f+L_{0} x\right)
\end{array}\right) \\
& =\left(\begin{array}{c}
A_{0} f-L_{0} B f-L_{0} N x \\
B f+N x
\end{array}\right) \\
& =\left(\begin{array}{cc}
G_{0} & -L_{0} N \\
B & N
\end{array}\right)\left(\begin{array}{l}
f \\
x
\end{array}\right) .
\end{aligned}
$$

Step 2. The operator $\mathcal{A}_{0}: D\left(\mathcal{A}_{0}\right) \subset X_{0} \rightarrow X_{0}$ given by

$$
\mathcal{A}_{0}:=\left(\begin{array}{cc}
G_{0} & -L_{0} N \\
0 & N
\end{array}\right), \quad D\left(\mathcal{A}_{0}\right):=D(\mathcal{A})
$$

generates an analytic semigroup of angle $\alpha>0$ on $x_{0}$. 
Proof. By assumption $A$ generates an analytic semigroup of angle $\alpha>0$ on $X$. Hence, by Step $1, \mathcal{A}$ generates an analytic semigroup of angle $\alpha>0$ on $X_{0}$. Since $B$ is relatively $A_{0^{-}}$ bounded with bound zero, a simple computation using the triangle inequality shows that $\mathcal{B}:=\left(\begin{array}{ll}0 & 0 \\ B & 0\end{array}\right)$ with domain $D(\mathcal{B}):=\left(D(B) \cap X_{0}\right) \times \partial X$ is relatively $\mathcal{A}$-bounded with bound zero. Hence, by [EN00, Lemma III.2.6] also $\mathcal{A}_{0}=\mathcal{A}-\mathcal{B}$ generates an analytic semigroup with angle $\alpha>0$ on $x_{0}$.

Step 3. There exists $\lambda_{0} \in \mathbb{R}$ such that $\left[\lambda_{0},+\infty\right) \subset \rho\left(G_{0}\right) \cap \rho\left(G_{00}\right) \cap \rho(N)$ and

$$
R\left(\lambda, \mathcal{A}_{0}\right)=\left(\begin{array}{cc}
R\left(\lambda, G_{00}\right) & -R\left(\lambda, G_{0}\right) L_{0} N R(\lambda, N) \\
0 & R(\lambda, N)
\end{array}\right) \quad \text { for } \lambda \geq \lambda_{0} .
$$

Proof. By assumption $A_{0}$ is a weak Hille-Yosida operator. Since $A_{0}$ and $G_{0}=A_{0}-L_{0} B$ differ only by a relatively bounded perturbation of bound 0 , by [EN00, Lem. III.2.5] also $G_{0}$ is a weak Hille-Yosida operator. In particular, there exists $\lambda_{0} \in \mathbb{R}$ such that $\left[\lambda_{0},+\infty\right) \subset \rho\left(G_{0}\right) \cap \rho\left(\mathcal{A}_{0}\right)$. Moreover, [EN00, Prop. IV.2.17] implies $\rho\left(G_{0}\right)=\rho\left(G_{00}\right)$ which shows the first claim.

Next we claim that $\lambda-N$ is injective for $\lambda \geq \lambda_{0}$. If by contradiction we assume that there exists $0 \neq x \in \operatorname{ker}(\lambda-N)$, a simple computation shows that

$$
0 \neq\left(\begin{array}{c}
-R\left(\lambda, G_{0}\right) L_{0} N x \\
x
\end{array}\right) \in \operatorname{ker}\left(\lambda-\mathcal{A}_{0}\right)
$$

contradicting the fact $\lambda \in \rho\left(\mathcal{A}_{0}\right)$. Let now $R\left(\lambda, \mathcal{A}_{0}\right)=\left(R_{i j}(\lambda)\right)_{2 \times 2}$ and choose some arbitrary $\left(\begin{array}{l}g \\ y\end{array}\right) \in X_{0}$. Then we have

$$
\begin{aligned}
\left(\begin{array}{l}
R_{11}(\lambda) g+R_{12}(\lambda) y \\
R_{21}(\lambda) g+R_{22}(\lambda) y
\end{array}\right)=\left(\begin{array}{l}
f \\
x
\end{array}\right) & \Longleftrightarrow\left(\lambda-\mathcal{A}_{0}\right)\left(\begin{array}{l}
f \\
x
\end{array}\right)=\left(\begin{array}{l}
g \\
y
\end{array}\right) \\
& \Longleftrightarrow \begin{cases}\left(\lambda-G_{0}\right) f+L_{0} N x & =g \\
(\lambda-N) x & =y \\
L G_{0} f & =N x .\end{cases}
\end{aligned}
$$

For $y=0$ it follows $(\lambda-N) x=0$ and hence $x=0$. This implies $R_{21}(\lambda)=0$. Moreover, by (3.2) the operator $\lambda-N$ must be surjective, hence it is invertible with inverse $(\lambda-N)^{-1}=$ $R_{22}(\lambda) \in \mathcal{L}(\partial X)$. Again by (3.2) this implies $R_{11}(\lambda)=R\left(\lambda, G_{00}\right)$. On the other hand, choosing $g=0$ we obtain $R_{21}(\lambda)=-R\left(\lambda, G_{0}\right) L_{0} N R(\lambda, N)$ as claimed.

Step 4. $D_{00}$ and $N$ generate analytic semigroups of angle $\alpha>0$ on $X_{0}$ and $\partial X$, respectively.

Proof. Denote by $\left(\mathcal{T}_{0}(t)_{t \geq 0}\right.$ the semigroup generated by $\mathcal{A}_{0}$. Then by [EN00, Thm. II.1.10] for $\lambda \in \mathbb{R}$ sufficiently large $\bar{R}\left(\lambda, \mathcal{A}_{0}\right)$ is given by the Laplace transform $\left(\mathcal{L T}_{0}(\bullet)\right)(\lambda)$ of $\left(\mathcal{T}_{0}(t)_{t \geq 0}\right.$. Since $\mathcal{L}$ is injective, (3.1) implies that the semigroup generated by $\mathcal{A}_{0}$ is given by

$$
\mathcal{T}_{0}(t)=\left(\begin{array}{cc}
T(t) & * \\
0 & S(t)
\end{array}\right)
$$

where $(T(t))_{t \geq 0}$ and $(S(t))_{t \geq 0}$ are semigroups on $X_{0}$ and $\partial X$ generated by $G_{00}$ and $N$, respectively. Since by assumption $\left(\mathcal{T}_{0}(t)_{t \geq 0}\right.$ is analytic of angle $\alpha>0$, also the semigroups generated by $G_{00}$ and $N$ are analytic of angle $\alpha$.

This completes the proof of Theorem 3.1.

Since by [EN00, Thm. II.4.29] an analytic semigroup is compact if and only if its generator has compact resolvent, the following result relates compactness of the semigroups generated by $A$ and $D_{00}, N$.

Corollary 3.2. Let $D \in\{A, G\}$. Then $A$ has compact resolvent if and only if $D_{0}$ and $N$ have compact resolvents on $X$ and $\partial X$, respectively. 
Proof. By Step 1, $A$ has compact resolvent if and only if $\mathcal{A}$ has. Since $\mathcal{A}$ and $\mathcal{A}_{0}$ differ only by the relatively bounded perturbation $\mathcal{B}:=\left(\begin{array}{cc}0 & 0 \\ B & 0\end{array}\right)$ of bound 0 , by [EN00, III- $\left.(2.5)\right]$ one of the operators $\mathcal{A}, \mathcal{A}_{0}$ has compact resolvent if and only if the other has. Let $\lambda \in \rho\left(\mathcal{A}_{0}\right)$. Then by (3.1) $R\left(\lambda, \mathcal{A}_{0}\right)$ is compact if and only if $R\left(\lambda, G_{00}\right), R(\lambda, N)$ and

$$
-R\left(\lambda, G_{0}\right) L_{0} N R(\lambda, N)=R\left(\lambda, G_{0}\right) L_{0}-\lambda R\left(\lambda, G_{0}\right) L_{0} R(\lambda, N)
$$

are all compact. The latter is the case if and only if $R\left(\lambda, G_{0}\right) L_{0}$ is compact. Now writing

$$
R\left(\lambda, G_{0}\right)=R\left(\lambda, G_{00}\right) \cdot\left(\operatorname{Id}-L_{0} L\right)+R\left(\lambda, G_{0}\right) L_{0} \cdot L
$$

we conclude that $R\left(\lambda, \mathcal{A}_{0}\right)$ is compact if and only if $R\left(\lambda, G_{0}\right)$ and $R(\lambda, N)$ are compact.

\section{Perturbations of Operators with Generalized Wentzell Boundary CONDITIONS}

In many applications the feedback operator $B: D(B) \subset X \rightarrow \partial X$ which determines the boundary condition in (2.1) splits into a sum

$$
B=B_{0}+C L, \quad D(B)=D\left(B_{0}\right) \cap D(C L)
$$

for some $C: D(C) \subset \partial X \rightarrow \partial X$. For example in (1.1) we could choose $B_{0}=\beta \frac{\partial}{\partial n}$ (which determines the feedback from the interior of $\Omega$ to the boundary $\partial \Omega$ ) and the multiplication operator $C=M_{\gamma} \in \mathcal{L}(\partial X)$ (which governs the "free" evolution on $\partial \Omega$ ). Next we study this situation in more detail where we allow $C$ to be unbounded. For a concrete example see $\left[\mathrm{FGG}^{+} 10,(1.2),(3.3)\right]$ and Subsection 5.3. Moreover, we will introduce a relatively bounded perturbation $P$ of the operator $A_{m}$.

To this end we first have to generalize our notation concerning the Dirichlet- and Dirichlet-toNeumann operators. For a closed operator $D_{m}: D\left(D_{m}\right) \subset X \rightarrow X$ let $D_{0} \subset D_{m}$ with domain $D\left(D_{0}\right):=D\left(D_{m}\right) \cap \operatorname{ker}(L)$ on $X$. Then by [Gre87, Lem. 1.2] for $\lambda \in \rho\left(D_{0}\right)$ the restriction $\left.L\right|_{\operatorname{ker}\left(\lambda-D_{m}\right)}: \operatorname{ker}\left(\lambda-D_{m}\right) \rightarrow \partial X$ is invertible with bounded inverse

$$
L_{\lambda}^{D_{m}}:=\left(\left.L\right|_{\operatorname{ker}\left(\lambda-D_{m}\right)}\right)^{-1}: \partial X \rightarrow \operatorname{ker}\left(\lambda-D_{m}\right) \subseteq X,
$$

which we call the abstract Dirichlet operator associated to $\lambda$ and $D_{m}$. Note that $L_{\lambda}^{D_{m}}=$ $L_{0}^{D_{m}-\lambda}$, that is $L_{\lambda}^{D_{m}} x=f$ gives the unique solution of the abstract Dirichlet problem

$$
\left\{\begin{array}{c}
D_{m} f=\lambda f, \\
L f=x .
\end{array}\right.
$$

If $D_{m}=A_{m}$ we will simply write $L_{\lambda}:=L_{\lambda}^{A_{m}}$.

Next, for a relatively $D_{0}$-bounded feedback operator $F: D(F) \subset X \rightarrow \partial X$ we introduce the associated generalized abstract Dirichlet-to-Neumann operator $N_{\lambda}^{D_{m}, F}: D\left(N_{\lambda}^{D_{m}, F}\right) \subset \partial X \rightarrow$ $\partial X$ defined by

$$
N_{\lambda}^{D_{m}, F} x:=F L_{\lambda}^{D_{m}} x, \quad D\left(N_{\lambda}^{D_{m}, F}\right):=\left\{x \in \partial X: L_{\lambda}^{D_{m}} x \in D(F)\right\} .
$$

If $\lambda=0$ we simply write $N^{D_{m}, F}:=N_{0}^{D_{m}, F}$. If in addition $F=B$ we put $N^{D_{m}}:=N_{0}^{D_{m}, B}$ and $N^{F}:=N_{0}^{A_{m}, F}$ in case $D_{m}=A_{m}$. Finally, as before we set $N:=N_{0}^{A_{m}, B}$.

To proceed we need the following domain inclusions where $B, B_{0}: D(B) \subset X \rightarrow \partial X$ are relatively $A_{0}$-bounded and $C: D(C) \subset \partial X \rightarrow \partial X$.

Lemma 4.1. The following assertions hold true.

(i) If $C$ is relatively $N^{B_{0}}$-bounded, then $D\left(B_{0}\right) \subseteq D(C L)$.

(ii) If $N^{B_{0}}$ is relatively $C$-bounded, then $D\left(A_{m}\right) \cap D(C L) \subseteq D\left(B_{0}\right)$. 
Proof. (i). Recall that $L_{0}: \partial X \rightarrow \operatorname{ker}\left(A_{m}\right)$ is bijective with inverse $L$. Hence, using the first decomposition in (2.2) we conclude

$$
\begin{aligned}
L D\left(B_{0}\right) & =L\left(\left(X_{0} \oplus \operatorname{ker}\left(A_{m}\right)\right) \cap D\left(B_{0}\right)\right) \\
& =L\left(\operatorname{ker}\left(A_{m}\right) \cap D\left(B_{0}\right)\right) \\
& =L_{0}^{-1}\left(\operatorname{ker}\left(A_{m}\right) \cap D\left(B_{0}\right)\right) \\
& \subseteq D\left(N^{B_{0}}\right) \subseteq D(C) .
\end{aligned}
$$

This implies the claim.

(ii). By assumption, we have

$$
L D(C L) \subseteq D(C) \subseteq D\left(N^{B_{0}}\right)
$$

This implies

$$
L_{0} L D(C L) \subseteq L_{0} D\left(N^{B_{0}}\right) \subseteq D\left(B_{0}\right) .
$$

On the other hand, $\left(\mathrm{Id}-L_{0} L\right) D\left(A_{m}\right)=D\left(A_{0}\right) \subseteq D\left(B_{0}\right)$. Summing up this gives the desired inclusion.

Note that in part (ii) of the previous result we cannot expect the inclusion $D(C L) \subset D\left(B_{0}\right)$ since always $X_{0}=\operatorname{ker}(L) \subset D(C L)$ holds.

We now return to the decomposition $B=B_{0}+C L$ from (4.1) and consider for a relatively $A_{m^{-}}$ bounded perturbation $P: D(P) \subset X \rightarrow X$ the operator $(A+P)^{B}: D\left((A+B)^{P}\right) \subseteq X \rightarrow X$ given by

$$
\begin{aligned}
(A+P)^{B} & \subseteq A_{m}+P, \\
D\left((A+P)^{B}\right) & :=\left\{f \in D\left(A_{m}\right) \cap D\left(B_{0}\right) \cap D(C L): L A_{m} f+P f=B_{0} f+C L f\right\} .
\end{aligned}
$$

Next we assume that $C$ is relatively $N^{B_{0}}=B_{0} L_{0}^{A_{m}}$-bounded of bound 0 . Note that by the previous lemma part (i) this implies that $D(B)=D\left(B_{0}\right) \cap D(C L)=D\left(B_{0}\right)$.

Theorem 4.2. Let $P: D(P) \subset X \rightarrow X$ be relatively $A_{m}$-bounded with $A_{0}$-bound 0 and let $C: D(C) \subset \partial X \rightarrow \partial X$ be relatively $N^{B_{0}}$-bounded of bound 0 . Then for $B$ given by (4.1) the following statements are equivalent.

(a) $(A+P)^{B}$ in (4.2) generates an analytic semigroup of angle $\alpha>0$ on $X$.

(b) $A^{B_{0}}$ generates an analytic semigroup of angle $\alpha>0$ on $X$.

(c) $A_{0}$ is sectorial of angle $\alpha>0$ on $X$ and $N^{B_{0}}$ generates an analytic semigroup of angle $\alpha>0$ on $\partial X$.

Before giving the proof we state an analogous result where we interchange the roles of $N^{B_{0}}$ and $C$. That is, we assume that $N^{B_{0}}$ is relatively $C$-bounded of bound 0 . Note that by Lemma 4.1.(ii) this implies that $D\left(A_{m}\right) \cap D(B)=D\left(A_{m}\right) \cap D\left(B_{0}\right) \cap D(C L)=D\left(A_{m}\right) \cap D(C L)$.

Theorem 4.3. Let $P: D(P) \subset X \rightarrow X$ be relatively $A_{m}$-bounded with $A_{0}$-bound 0 and let $N^{B_{0}}$ be relatively $C$-bounded of bound 0 for some $C: D(C) \subset \partial X \rightarrow \partial X$. Then for $B$ given by (4.1) the following statements are equivalent.

(a) $(A+P)^{B}$ in $(4.2)$ generates an analytic semigroup of angle $\alpha>0$ on $X$.

(b) $A^{C L}$ generates an analytic semigroup of angle $\alpha>0$ on $X$.

(c) $A_{0}$ is sectorial of angle $\alpha>0$ on $X$ and $C$ generates an analytic semigroup of angle $\alpha>0$ on $\partial X$.

To prove the previous two theorems we use a series of auxiliary results. First we show the equivalences of (a) and (b) in case $P=0$.

Lemma 4.4. Let $C: D(C) \subset \partial X \rightarrow \partial X$ be relatively $N^{B_{0}}$-bounded of bound 0 . Then the following statements are equivalent. 
(a) $A^{B_{0}}$ generates an analytic semigroup of angle $\alpha>0$ on $X$.

(b) $A^{B}$ generates an analytic semigroup of angle $\alpha>0$ on $X$.

Proof. By Lemma 4.1.(i) the operator

$$
B:=B_{0}+C L, \quad D(B)=D\left(B_{0}\right)
$$

is well-defined. Since $D\left(A_{0}\right) \subset X_{0}$, the operators $B$ and $B_{0}$ coincide on $D\left(A_{0}\right)$. Hence, $B$ is relatively $A_{0}$-bounded if and only if $B_{0}$ is relatively $A_{0}$-bounded of bound 0 . Moreover, we have

$$
N^{B}=B L_{0}=N^{B_{0}}+C, \quad D\left(N^{B}\right)=D\left(N^{B_{0}}\right) .
$$

By [EN00, Thm. III.2.10] it then follows that $N^{B}$ generates an analytic semigroup of angle $\alpha>0$ on $\partial X$ if and only if $N^{B_{0}}$ does. The claim now follows by Theorem 3.1.

Lemma 4.5. Let $N^{B_{0}}$ be relatively $C$-bounded of bound 0 for some $C: D(C) \subset \partial X \rightarrow \partial X$. Then the following statements are equivalent.

(a) $A^{C L}$ generates an analytic semigroup of angle $\alpha>0$ on $X$.

(b) $A^{B}$ generates an analytic semigroup of angle $\alpha>0$ on $X$.

Proof. Let

$$
B:=B_{0}+C L, \quad D(B)=D\left(B_{0}\right) \cap D(C L) .
$$

By the same reasoning as in the previous proof we conclude that $B$ is relatively $A_{0}$-bounded if and only if $B_{0}$ is relatively $A_{0}$-bounded of the same bound 0. Moreover, by Lemma 4.1.(ii) we have

$$
\begin{aligned}
x \in D\left(N^{B}\right) & \Longleftrightarrow \quad L_{0} x \in D(B) \\
& \Longleftrightarrow \quad L_{0} x \in D\left(B_{0}\right) \cap D(C L) \cap D\left(A_{m}\right) \\
& \Longleftrightarrow \quad L_{0} x \in D(C L) \cap D\left(A_{m}\right) \\
& \Longleftrightarrow \quad L_{0} x \in D(C L) \\
& \Longleftrightarrow x \in L D(C L) \subseteq D(C) .
\end{aligned}
$$

This implies

$$
N^{B}=B L_{0}=N^{B_{0}}+C, \quad D\left(N^{B}\right)=D(C) .
$$

By [EN00, Thm. III.2.10] it follows that $N^{B}$ generates an analytic semigroup of angle $\alpha>0$ on $\partial X$ if and only if $C$ does. The claim then follows by Theorem 3.1.

Next we study how Dirichlet operators behave under perturbations.

Lemma 4.6. Let $P: D(P) \subset X \rightarrow X$ be a relatively $A_{m}$-bounded perturbation. Then for $\lambda \in \rho\left(A_{0}\right) \cap \rho\left(A_{0}+P\right)$ the Dirichlet operator $L_{\lambda}^{A_{m}+P} \in \mathcal{L}(\partial X, X)$ exists and satisfies

$$
L_{\lambda}^{A_{m}+P}-L_{\lambda}^{A_{m}}=R\left(\lambda, A_{0}+P\right) P L_{\lambda}^{A_{m}}=R\left(\lambda, A_{0}\right) P L_{\lambda}^{A_{m}+P} .
$$

Proof. Let $\left[D\left(A_{m}\right)\right]:=\left(D\left(A_{m}\right),\|\cdot\|_{A_{m}}\right)$ for the graph norm $\|\cdot\|_{A_{m}}:=\|\cdot\|_{X}+\left\|A_{m} \cdot\right\|_{X}$. Then $P:\left[D\left(A_{m}\right)\right] \rightarrow X$ and $L_{\lambda}^{A_{m}}: \partial X \rightarrow\left[D\left(A_{m}\right)\right]$ are bounded, hence $P L_{\lambda}^{A_{m}}: \partial X \rightarrow X$ is bounded as well. This implies that

$$
T:=L_{\lambda}^{A_{m}}+R\left(\lambda, A_{0}+P\right) P L_{\lambda}^{A_{m}} \in \mathcal{L}(\partial X, X) .
$$

Since

$$
\begin{aligned}
\left(A_{m}+P-\lambda\right) T x & =\left(A_{m}+P-\lambda\right) L_{\lambda}^{A_{m}} x+\left(A_{m}+P-\lambda\right) R\left(\lambda, A_{0}+P\right) P L_{\lambda}^{A_{m}} \\
& =P L_{\lambda}^{A_{m}} x-P L_{\lambda}^{A_{m}} x=0,
\end{aligned}
$$

we have $\operatorname{rg}(T) \subseteq \operatorname{ker}\left(\lambda-A_{m}-P\right)$. Moreover, from

$$
\operatorname{rg}\left(R\left(\lambda, A_{0}+P\right) P L_{\lambda}^{A_{m}}\right) \subset D\left(A_{0}+P\right)=D\left(A_{0}\right) \subset \operatorname{ker}(L)
$$


it follows that $L T x=L L_{\lambda}^{A_{m}} x=x$. Hence, $\left.L\right|_{\operatorname{ker}\left(\lambda-A_{m}-P\right)}$ is surjective with right-inverse $T$. Since $\operatorname{ker}\left(\lambda-A_{m}-P\right) \cap X_{0} \subset \operatorname{ker}\left(\lambda-A_{0}-P\right)=\{0\}$ we conclude that $\left.L\right|_{\operatorname{ker}\left(\lambda-A_{m}-P\right)}$ is injective as well. This implies that it is invertible with inverse $L_{\lambda}^{A_{m}+P}=T$ and proves the first identity in (4.3). The second one follows by changing the roles of $A_{m}$ and $A_{m}+P$.

Next we consider perturbations of Dirichlet-to-Neumann operators.

Proposition 4.7. Let $P: D(P) \subset X \rightarrow X$ be a relatively $A_{m}$-bounded perturbation. Then for $\lambda \in \rho\left(A_{0}\right) \cap \rho\left(A_{0}+P\right)$ the perturbed Dirichlet-to-Neumann operator $N_{\lambda}^{A_{m}+P}$ exists, $D\left(N_{\lambda}^{A_{m}}\right)=$ $D\left(N_{\lambda}^{A_{m}+P}\right)$ and the difference $N_{\lambda}^{A_{m}}-N_{\lambda}^{A_{m}+P}$ is bounded.

Proof. Since

$$
\operatorname{rg}\left(R\left(\lambda, A_{0}\right)\left(A_{m}-\lambda\right) L_{\lambda}^{A_{m}+P}\right) \subset D\left(A_{0}\right) \subset D(B),
$$

by Lemma 4.6 it follows that $D\left(N_{\lambda}^{A_{m}}\right)=D\left(N_{\lambda}^{A_{m}+P}\right)$. Moreover, from (4.3) we conclude

$$
N_{\lambda}^{A_{m}}-N_{\lambda}^{A_{m}+P}=B L_{\lambda}^{A_{m}}-B L_{\lambda}^{A_{m}+P} \supseteq-B R\left(\lambda, A_{0}\right) P L_{\lambda}^{A_{m}+P} \in \mathcal{L}(\partial X) .
$$

To conclude the proofs of Theorem 4.2 and Theorem 4.3, we need one further result. It shows that the assertion (a) in both results is independent under the perturbation $P$.

Lemma 4.8. Let $P: D(P) \subset X \rightarrow X$ relatively $A_{m}$-bounded with $A_{0}$-bound 0 . Then the following statements are equivalent.

(a) $A^{B}$ generates an analytic semigroup of angle $\alpha>0$ on $X$.

(b) $(A+P)^{B}$ generates an analytic semigroup of angle $\alpha>0$ on $X$.

Proof. Since $A_{0}$ is a weak Hille-Yosida operator and $P$ is relatively $A_{0}$-bounded of bound 0 , by [EN00, Lem. III.2.6] there exists a $\lambda \in \rho\left(A_{0}\right) \cap \rho\left(A_{0}+P\right)$ and $A_{0}-\lambda, A_{0}+P-\lambda$ are again weak Hille-Yosida operators. Since $B$ is relatively $A_{0}$-bounded of bound 0 a simple computation shows that it is also relatively $\left(A_{0}-\lambda\right)$ - and $\left(A_{0}+P-\lambda\right)$-bounded of bound 0 . Moreover, by Lemma 4.6 the operators $L_{0}^{A_{m}-\lambda}$ and $L_{0}^{A_{m}+P-\lambda}$ exist and are bounded. Hence, $A_{0}-\lambda$ and $A_{0}+P-\lambda$ both satisfy Assumptions 2.2.

Next we check the conditions in Theorem 3.1. By [EN00, Lem. III.2.6] the operator $A_{0}-\lambda$ is sectorial of angle $\alpha>0$ on $X$ if and only if $A_{0}+P-\lambda$ is. Moreover, by Proposition 4.7 $N^{A_{m}-\lambda}$ generates an analytic semigroup of angle $\alpha>0$ if and only if $N^{A_{m}+P-\lambda}$ does. Applying Theorem 3.1 to $A_{0}-\lambda, N^{A_{m}-\lambda}$ and $A_{0}+P-\lambda, N^{A_{m}+P-\lambda}$, respectively, the claim follows.

Proof of Theorem 4.2 and Theorem 4.3. By Lemma 4.8 assertion (a) is independent of $P$ while by Lemma 4.4 and Lemma 4.5, respectively, for $P=0$ it is equivalent to (b). Since the equivalence of (b) and (c) follows Theorem 3.1 the proof is complete.

\section{EXAMPLES}

5.1. Second Order Differential Operators on $C\left([\mathbf{0}, \mathbf{1}], \mathbb{C}^{\mathbf{n}}\right)$. For $n \in \mathbb{N}$ consider functions $a_{i} \in \mathrm{C}[0,1] \cap \mathrm{C}^{1}(0,1), 1 \leq i \leq n$, being strictly positive on $(0,1)$ such that $\frac{1}{a_{i}} \in L^{1}[0,1]$. Let $a:=\operatorname{diag}\left(a_{1}, \ldots, a_{n}\right)$ and $b, c \in \mathrm{C}\left([0,1], \mathrm{M}_{n}(\mathbb{C})\right)$. Moreover, define the maximal operator $A_{m}: D\left(A_{m}\right) \subset \mathrm{C}\left([0,1], \mathbb{C}^{n}\right) \rightarrow \mathrm{C}\left([0,1], \mathbb{C}^{n}\right)$ by

$$
A_{m}:=a f^{\prime \prime}+b f^{\prime}+c f, \quad D\left(A_{m}\right):=\left\{f \in \mathrm{C}\left([0,1], \mathbb{C}^{n}\right) \cap \mathrm{C}^{2}\left((0,1), \mathbb{C}^{n}\right): A_{m} f \in \mathrm{C}\left([0,1], \mathbb{C}^{n}\right)\right\}
$$

and take $B \in \mathcal{L}\left(C^{1}\left([0,1], \mathbb{C}^{n}\right), \mathbb{C}^{2 n}\right)$.

Corollary 5.1. We have $D\left(A_{m}\right) \subset \mathrm{C}^{1}\left([0,1], \mathbb{C}^{n}\right)=D(B)$ and

$$
A \subseteq A_{m}, \quad D(A)=\left\{f \in D\left(A_{m}\right):\left(\begin{array}{l}
\left(A_{m} f\right)(0) \\
\left(A_{m} f\right)(1)
\end{array}\right)=B f\right\}
$$

generates a compact and analytic semigroup of angle $\frac{\pi}{2}$ on $\mathrm{C}\left([0,1], \mathbb{C}^{n}\right)$. 
Proof. We consider $X:=\mathrm{C}\left([0,1], \mathbb{C}^{n}\right)=\mathrm{C}[0,1] \times \cdots \times \mathrm{C}[0,1]$ equipped with the norm $\|f\|_{1, \infty}:=\left\|f_{1}\right\|_{\infty}+\cdots+\left\|f_{n}\right\|_{\infty}, \partial X:=\mathbb{C}^{2 n}$ and define $L \in \mathcal{L}(X, \partial X)$ by $L f:=\left(\begin{array}{l}f(0) \\ f(1)\end{array}\right)$. Then as in [EF05, Cor. 4.1 Step (iii)] it follows that $D\left(A_{m}\right) \subset D(B)$, hence $A$ coincides with the operator defined in (2.1). Since

$$
\text { Pf }:=b f^{\prime}+c f, \quad D(P):=\mathrm{C}^{1}\left([0,1], \mathbb{C}^{n}\right)
$$

is a relatively $A_{m}$-bounded with $A_{0}$-bound 0 (see Step 4 below), we assume by Theorem 4.2 without loss of generality that $b=c=0$.

Next we verify Assumptions 2.2 and the hypotheses of Theorem 3.1.

Step 1. The abstract Dirichlet operator $L_{0} \in \mathcal{L}(\partial X, X)$ exists.

Proof. We have $\operatorname{ker}\left(A_{m}\right)=\operatorname{lin}\left\{\varepsilon_{0}, \varepsilon_{1}\right\}$ for

$$
\varepsilon_{0}(s):=1-s \text { and } \varepsilon_{1}(s):=s, s \in[0,1] .
$$

A simple calculation then shows that $L_{0}:=\left(\left.L\right|_{\operatorname{ker}\left(A_{m}\right)}\right)^{-1} \in \mathcal{L}(\partial X, X)$ is given by

$$
L_{0}\left(\begin{array}{c}
x_{1} \\
\vdots \\
x_{2 n}
\end{array}\right)=\varepsilon_{0} \cdot\left(\begin{array}{c}
x_{1} \\
\vdots \\
x_{n}
\end{array}\right)+\varepsilon_{1} \cdot\left(\begin{array}{c}
x_{n+1} \\
\vdots \\
x_{2 n}
\end{array}\right) \text {. }
$$

Step 2. The operator $A_{0}$ on $X$ is sectorial of angle $\frac{\pi}{2}$ and has compact resolvent.

Proof. Let $A_{i}:=a_{i} \cdot \frac{d^{2}}{d s^{2}}$ with domain $D\left(A_{i}\right):=\left\{g \in \mathrm{C}[0,1] \cap \mathrm{C}^{2}(0,1): a_{i} \cdot g^{\prime \prime} \in \mathrm{C}[0,1]\right\}$ for $1 \leq i \leq n$. Then

$$
R\left(\lambda, A_{0}\right)=\operatorname{diag}\left(R\left(\lambda, A_{1}\right), \ldots, R\left(\lambda, A_{n}\right)\right) .
$$

Since by [EF05, Cor. 4.1. Step (ii)] all $A_{i}$ are sectorial of angle $\frac{\pi}{2}$ and have compact resolvents on $\mathrm{C}[0,1]$, the claim follows.

Step 3. The maximal operator $A_{m}$ is densely defined and closed.

Proof. Since $\mathrm{C}^{2}\left([0,1], \mathbb{C}^{n}\right) \subset D\left(A_{m}\right), A_{m}$ is densely defined. By Step 1, Step 2 and [EF05, Lem. 3.2] it follows that $A_{m}$ is closed.

Step 4. The feedback operator $B$ is relatively $A_{0}$-bounded of bound 0 .

Proof. Since $D(B)=\mathrm{C}^{1}\left([0,1], \mathbb{C}^{n}\right)$ it suffices to show that the first derivative with domain $\mathrm{C}^{1}\left([0,1], \mathbb{C}^{n}\right)$ is relatively $A_{0}$-bounded with bound 0 . Let $f \in D\left(A_{0}\right)$. Then by [EF05, Cor. 4.1. Step (iii)] it follows that for all $\varepsilon>0$ there exists a constant $C_{\varepsilon}>0$ such that

$$
\begin{aligned}
\left\|f^{\prime}\right\|_{1, \infty} & \leq \varepsilon \cdot\left\|A_{1} f_{1}\right\|_{\infty}+\cdots+\varepsilon \cdot\left\|A_{n} f_{n}\right\|_{\infty}+C_{\varepsilon} \cdot\left\|f_{1}\right\|_{\infty}+\cdots+C_{\varepsilon} \cdot\left\|f_{n}\right\|_{\infty} \\
& =\varepsilon \cdot\left\|A_{0} f\right\|_{1, \infty}+C_{\varepsilon} \cdot\|f\|_{1, \infty} .
\end{aligned}
$$

Step 5. The Dirichlet-to-Neumann operator $N$ generates an analytic, compact semigroup of angle $\frac{\pi}{2}$ on $\partial X$.

Proof. Since the boundary space $\partial X$ is finite dimensional, $N$ is bounded. Hence $N$ generates an analytic, compact semigroup of angle $\frac{\pi}{2}$ on $\partial X$.

Summing up, by Theorem 3.1 and Corollary 3.2 the claim follows completing the proof.

Remark 5.2. Corollary 5.1 generalizes [EF05, Cor. 4.1] to arbitrary $n \in \mathbb{N}$.

We give a particular choice for the operator $B$.

Corollary 5.3. For $M_{i}, N_{i} \in \mathrm{M}_{2 n \times n}(\mathbb{C}), i=0,1$, the operator

$$
A \subseteq A_{m}, D(A)=\left\{f \in D\left(A_{m}\right):\left(\begin{array}{l}
\left(A_{m} f\right)(0) \\
\left(A_{m} f\right)(1)
\end{array}\right)=M_{0} f^{\prime}(0)+M_{1} f^{\prime}(1)+N_{0} f(0)+N_{1} f(1)\right\}
$$

generates a compact and analytic semigroup of angle $\frac{\pi}{2}$ on $\mathrm{C}\left([0,1], \mathbb{C}^{n}\right)$. 
We remark that second order differential operators on spaces of functions $f:[0,1] \rightarrow \mathbb{C}^{n}$ can be used to describe diffusion- and waves on networks. For some recent results in the $\mathrm{L}^{p}$-context for operators with generalized Robin-type boundary conditions we refer to [EK].

5.2. Banach Space-Valued Second Derivative. We associate to an arbitrary Banach space $Y$ the Banach space $X:=\mathrm{C}([0,1], Y)$ of all continuous functions on $[0,1]$ with values in $Y$ equipped with the sup-norm. Moreover, we take $\left.P \in \mathcal{L}\left(\mathrm{C}^{1}([0,1], Y), X\right)\right), \Phi \in \mathcal{L}\left(X, Y^{2}\right)$ and an operator $(\mathcal{C}, D(\mathcal{C}))$ on $Y^{2}$. Then the following holds.

Corollary 5.4. The operator $\mathcal{C}$ generates an analytic semigroups of angle $\alpha \in\left(0, \frac{\pi}{2}\right]$ on $Y^{2}$ if and only if the operator

$$
\begin{aligned}
A f & :=f^{\prime \prime}+P f \\
D(A) & :=\left\{f \in \mathrm{C}^{2}([0,1], Y):\left(\begin{array}{c}
f(0) \\
f(1)
\end{array}\right) \in D(\mathcal{C}),\left(\begin{array}{c}
f^{\prime \prime}(0)+P f(0) \\
f^{\prime \prime}(1)+P f(1)
\end{array}\right)=\Phi f+\mathcal{C}\left(\begin{array}{c}
f(0) \\
f(1)
\end{array}\right)\right\}
\end{aligned}
$$

generates an analytic semigroup of angle $\alpha \in\left(0, \frac{\pi}{2}\right]$ on $X$.

Proof. We consider $\partial X:=Y^{2}$ and define $L \in \mathcal{L}(X, \partial X)$ by $L f:=\left(\begin{array}{l}f(0) \\ f(1)\end{array}\right)$. Moreover, define

$$
A_{m}: D\left(A_{m}\right) \subseteq X \rightarrow X, \quad A_{m} f:=f^{\prime \prime}+P f, \quad D\left(A_{m}\right)=\mathrm{C}^{2}([0,1], Y)
$$

and

$$
B: D(B) \subseteq X \rightarrow \partial X, \quad B f:=\Phi f+\mathcal{C} L f, \quad D(B):=\left\{f \in X:\left(\begin{array}{l}
f(0) \\
f(1)
\end{array}\right) \in D(\mathcal{C})\right\} .
$$

Then $A$ coincides with the operator given by (2.1). Since $P$ is a relatively $A_{m}$-bounded of $A_{m}$-bound 0 and $\Phi \in \mathcal{L}(X, \partial X)$, by Theorem 4.3 it suffices to verify the Assumptions 2.2 and that $A_{0}$ is sectorial of angle $\alpha>0$.

Step 1. The abstract Dirichlet operator $L_{0} \in \mathcal{L}(\partial X, X)$ exists.

Proof. As in Step 1 of the proof of Corollary 5.1 we have $\operatorname{ker}\left(A_{m}\right)=\left\{\varepsilon_{0} y_{0}+\varepsilon_{1} y_{1}: y_{0}, y_{1} \in Y\right\}$ for

$$
\varepsilon_{0}(s):=1-s \text { and } \varepsilon_{1}(s):=s, s \in[0,1] .
$$

Moreover, $L_{0}:=\left(\left.L\right|_{\operatorname{ker}\left(A_{m}\right)}\right)^{-1} \in \mathcal{L}(\partial X, X)$ is given by

$$
L_{0}\left(\begin{array}{l}
y_{0} \\
y_{1}
\end{array}\right)=\varepsilon_{0} \cdot y_{0}+\varepsilon_{1} \cdot y_{1} .
$$

Step 2. The operator $A_{0}$ on $X$ is sectorial of angle $\frac{\pi}{2}$.

Proof. This follows as in the proof of [EN00, Thm VI. 4.1].

Step 3. The maximal operator $A_{m}$ is densely defined and closed.

Proof. Since $\mathrm{C}^{2}([0,1], Y) \subset D\left(A_{m}\right), A_{m}$ is densely defined. By Step 1, Step 2 and $[\mathrm{EF} 05$, Lem. 3.2] it follows that $A_{m}$ is closed.

Step 4. The feedback operator $B$ is relatively $A_{0}$-bounded of bound 0 .

Proof. For $f \in D\left(A_{0}\right) \subset X_{0}$ we have $B f=\Phi f$. Since $\Phi$ is bounded, this implies the claim.

Summing up, by Theorem 3.1 the claim follows completing the proof. 
5.3. Perturbations of the Laplacian on $\mathrm{C}(\bar{\Omega})$ with generalized Wentzell boundary conditions. In this subsection we complement the example from the introduction concerning the Laplacian on $\mathrm{C}(\bar{\Omega})$ with generalized Wentzell boundary conditions, see also [Eng03].

To this end we consider a bounded domain $\Omega \subset \mathbb{R}^{n}$ with $\mathrm{C}^{\infty}$-boundary $\partial \Omega$ and take an operator $P \in \mathcal{L}\left(\mathrm{C}^{1}(\bar{\Omega}), \mathrm{C}(\bar{\Omega})\right)$ (e.g. a first-order differential operator). Then we define the perturbed Laplacian $A: D(A) \subset \mathrm{C}(\bar{\Omega}) \rightarrow \mathrm{C}(\bar{\Omega})$ with generalized Wentzell boundary conditions by $A f:=\Delta_{m} f+P f$ for

$$
f \in D(A) \quad:\left.\Longleftrightarrow \quad\left(\Delta_{m} f+P f\right)\right|_{\partial \Omega}=\beta \cdot \frac{\partial}{\partial n} f+\left.\gamma \cdot f\right|_{\partial \Omega}+\left.q \cdot \Delta_{\Gamma} f\right|_{\partial \Omega},
$$

cf. also $\left[\mathrm{FGG}^{+} 10,(1.2),(3.3)\right]$. Here $\beta<0, \gamma \in \mathrm{C}(\partial \Omega), q \geq 0$ and $\Delta_{\Gamma}: D\left(\Delta_{\Gamma}\right) \subset \mathrm{C}(\partial \Omega) \rightarrow$ $\mathrm{C}(\partial \Omega)$ denotes the Laplace-Beltrami operator. In case $P=0, q=0$ this just gives the operator $A$ from the introduction. As we will see below for $q>0$ the Laplace-Beltrami operator will dominate the dynamic on the boundary $\partial X .{ }^{1}$ However, in this case essentially the same generation result holds as for $q=0$.

Corollary 5.5. For all $q>0$ the operator $A \subseteq \Delta_{m}+P$ with domain given in (5.1) generates a compact and analytic semigroup of angle $\frac{\pi}{2}$.

Proof. Without loss of generality we assume that $\beta=1$. To fit the operator $A$ into our setting we define $X:=\mathrm{C}(\bar{\Omega}), \partial X:=\mathrm{C}(\partial \Omega)$ and the trace $L \in \mathcal{L}(X, \partial X), L f:=\left.f\right|_{\partial \Omega}$. Then we consider $A_{m}:=\Delta_{m}: D\left(\Delta_{m}\right) \subset X \rightarrow X$ and $B_{0}:=\frac{\partial}{\partial n}: D\left(\frac{\partial}{\partial n}\right) \subset X \rightarrow \partial X$ as in [Eng03] and put $C:=q \cdot \Delta_{\Gamma}+M_{\gamma}: D\left(\Delta_{\Gamma}\right) \subset \partial X \rightarrow \partial X$ and $B:=B_{0}+C L$ as in (4.1).

Then by [ABHN01, Thm. 6.1.3], $A_{0}=\Delta_{0}$ is sectorial of angle $\frac{\pi}{2}$ and by [Eng03, (1.9)] and [EN00, Prop. II.4.25] has compact resolvent. Moreover, $C$ generates a compact analytic semigroup of angle $\frac{\pi}{2}$. Let $W:=\left(-\Delta_{\Gamma}\right)^{\frac{1}{2}}$. Then by the proof of [Eng03, Thm. 2.1] there exists a relatively $W$-bounded perturbation $Q: D(Q) \subset \partial X \rightarrow \partial X$ such that $N^{B_{0}}=B_{0} L_{0}^{A_{m}}=$ $-W+Q$. This implies that $N^{B_{0}}$ is relatively $W$-bounded and by [Paz83, Thm. 6.10] it follows that $N^{B_{0}}$ is relatively $C$-bounded of bound 0. Hence, by Theorem 4.3, $(A+P)^{B}$ generates an analytic semigroup of angle $\frac{\pi}{2}$. Compactness of this semigroup follows by Corollary 3.2.

We remark that Corollary 5.5 confirms the conjecture $\theta_{\infty}=\frac{\pi}{2}$ in $\left[\mathrm{FGG}^{+} 10\right.$, Sect. 5] for $a(x) \equiv \mathrm{Id}$ and constant $\beta<0$.

5.4. Uniformly Elliptic Operators on $\mathrm{C}(\overline{\boldsymbol{\Omega}})$. We consider a uniformly elliptic second-order differential operator with generalized Wentzell boundary conditions on $\mathrm{C}(\bar{\Omega})$ for a bounded domain $\Omega \subset \mathbb{R}^{n}$ with $\mathrm{C}^{\infty}$-boundary $\partial \Omega$. To this end, we first take real-valued functions

$$
a_{j k}=a_{k j} \in \mathrm{C}^{\infty}(\bar{\Omega}), \quad a_{j}, a_{0}, b_{0} \in \mathrm{C}(\bar{\Omega}), \quad 1 \leq j, k \leq n
$$

satisfying the uniform ellipticity condition

$$
\sum_{j, k=1}^{n} a_{j k}(x) \cdot \xi_{j} \xi_{k} \geq c \cdot\|\xi\|^{2} \quad \text { for all } x \in \bar{\Omega}, \xi=\left(\xi_{1}, \ldots, \xi_{n}\right) \in \mathbb{R}^{n}
$$

and some fixed $c>0$. Then we define the maximal operator $A_{m}: D\left(A_{m}\right) \subseteq \mathrm{C}(\bar{\Omega}) \rightarrow \mathrm{C}(\bar{\Omega})$ in divergence form by

$$
\begin{aligned}
A_{m} f & :=\sum_{j=1}^{n} \partial_{j}\left(\sum_{k=1}^{n} a_{j k} \partial_{k} f\right)+\sum_{k=1}^{n} a_{k} \partial_{k} f+a_{0} f, \\
D\left(A_{m}\right) & :=\left\{f \in \bigcap_{p \geq 1} W_{\mathrm{loc}}^{2, p}(\Omega): A_{m} f \in \mathrm{C}(\bar{\Omega})\right\},
\end{aligned}
$$

\footnotetext{
${ }^{1}$ The discussion of this case has been inspired by a discussion with J. Goldstein.
} 
and the feedback operator $B: D(B) \subseteq \mathrm{C}(\bar{\Omega}) \rightarrow \mathrm{C}(\partial \Omega)$ by

$$
B:=-\sum_{j, k=1}^{n} a_{j k} \nu_{j} L \partial_{k}+b_{0} L, \quad D(B):=\left\{f \in \bigcap_{p \geq 1} W_{\mathrm{loc}}^{2, p}(\Omega): B f \in \mathrm{C}(\partial \Omega)\right\},
$$

where $L \in \mathcal{L}(\mathrm{C}(\bar{\Omega}), \mathrm{C}(\partial \Omega)), L f:=\left.f\right|_{\partial \Omega}$ denotes the trace operator.

Corollary 5.6. The operator $A: D(A) \subseteq \mathrm{C}(\bar{\Omega}) \rightarrow \mathrm{C}(\bar{\Omega})$ given by

$$
A \subseteq A_{m}, \quad D(A):=\left\{f \in D\left(A_{m}\right) \cap D(B): L A_{m} f=B f\right\}
$$

generates a compact and analytic semigroup on $\mathrm{C}(\bar{\Omega})$.

Proof. Let $X:=\mathrm{C}(\bar{\Omega}), \partial X:=\mathrm{C}(\partial \Omega)$ and define the maximal operator $\tilde{A}_{m}: D\left(\tilde{A}_{m}\right) \subseteq X \rightarrow X$ by

$$
\tilde{A}_{m}:=\sum_{j=1}^{n} \partial_{j}\left(\sum_{k=1}^{n} a_{j k} \partial_{k}\right), \quad D\left(\tilde{A}_{m}\right):=D\left(A_{m}\right),
$$

and the feedback operator $\tilde{B}: D(\tilde{B}) \subseteq \mathrm{C}(\bar{\Omega}) \rightarrow \mathrm{C}(\partial \Omega)$ by

$$
\tilde{B}:=-\sum_{j, k=1}^{n} a_{j k} \nu_{j} L \partial_{k}, \quad D(\tilde{B}):=\left\{f \in \bigcap_{p \geq 1} W_{\mathrm{loc}}^{2, p}(\Omega): \tilde{B} f \in \mathrm{C}(\partial \Omega)\right\} .
$$

Then by [EF05, Cor. 4.5] it follows that the operator $\tilde{A}: D(\tilde{A}) \subseteq X \rightarrow X$ with generalized Wentzell boundary conditions given by

$$
\tilde{A} \subseteq \tilde{A}_{m}, \quad D(\tilde{A}):=\left\{f \in D\left(\tilde{A}_{m}\right) \cap D(\tilde{B}): L \tilde{A}_{m} f=\tilde{B} f\right\}
$$

generates a compact and analytic semigroup on $X$. Let $P f:=\sum_{j=1}^{n} a_{j} \partial_{j} f+a_{0} f$ and $C f:=$ $b_{0} f$. Then $P$ is relatively $A_{m}$-bounded with bound 0 and $C \in \mathcal{L}(\partial X)$, hence the claim follows from Theorem 4.2 .

Remark 5.7. This result generalizes [EF05, Cor. 4.5] and via Theorem 3.1 also the main theorem in [Esc94]. Moreover, it shows that the angle of the analytic semigroup generated by $A$ only depends on the matrix $\left(a_{j k}\right)_{n \times n}$.

\section{Conclusion}

Our abstract approach allows to decompose an operator $A$ with generalized Wentzell boundary conditions into an operator $A_{0}$ with (much simpler) abstract Dirichlet boundary conditions and the associated abstract Dirichlet-to-Neumann operator $N$. In particular we prove, under a weak resolvent condition on $A_{0}$, that

$$
\left.\begin{array}{c}
A \text { generates an analytic semigroup } \\
\text { of angle } \alpha>0
\end{array}\right\} \Longleftrightarrow\left\{\begin{array}{l}
A_{0} \text { is sectorial of angle } \alpha>0, \text { and } \\
N \text { generates an analytic semigroup } \\
\text { of angle } \alpha>0,
\end{array}\right.
$$

cf. Theorem 3.1. This equivalence is new and shows the sharpness of our approach. Moreover, while being very general, our theory applied to concrete examples (where typically $A_{0}$ is wellunderstood and sectorial of angle $\frac{\pi}{2}$ ) gives new or improves known generation results, see Section 5 . 


\section{REFERENCES}

[ABHN01] W. Arendt, C. J. K. Batty, M. Hieber, and F. Neubrander. Vector-Valued Laplace Transforms and Cauchy Problems, Monographs in Mathematics, vol. 96. Birkhäuser (2001). http://www. springer.com/birkhauser/mathematics/book/978-3-0348-0086-0.

[CM98] M. Campiti and G. Metafune. Ventcel's boundary conditions and analytic semigroups. Arch. Math. 70 (1998), 377-390. http://dx.doi.org/10.1007/s000130050210.

[EF05] K.-J. Engel and G. Fragnelli. Analyticity of semigroups generated by operators with generalized Wentzell boundary conditions. Adv. Differential Equations 10 (2005), 1301-1320. http://projecteuclid.org/euclid.ade/1355867753.

[EK] K.-J. Engel and M. Kramar Fijavž. Waves and diffusion on metric graphs with general vertex conditions http://arxiv.org/pdf/1712.03030v1.

[EN00] K.-J. Engel and R. Nagel. One-Parameter Semigroups for Linear Evolution Equations, Graduate Texts in Math., vol. 194. Springer (2000). http://dx.doi.org/10.1007/b97696.

[Eng98] K.-J. Engel. Matrix representation of linear operators on product spaces. Rend. Circ. Mat. Palermo (2) Suppl. (1998), 219-224. International Workshop on Operator Theory (Cefalù, 1997).

[Eng99] K.-J. Engel. Spectral theory and generator property for one-sided coupled operator matrices. Semigroup Forum 58 (1999), 267-295. http://dx.doi.org/10.1007/s002339900020.

[Eng03] K.-J. Engel. The Laplacian on $C(\bar{\Omega})$ with generalized Wentzell boundary conditions. Arch. Math. 81 (2003), 548-558. http://dx.doi.org/10.1007/s00013-003-0557-y.

[Esc94] J. Escher. The Dirichlet-Neumann operator on continuous functions. Ann. Scuola Norm. Sup. Pisa Cl. Sci. (4) 21 (1994), 235-266. http://www.numdam.org/item?id=ASNSP_1994_4_21_2_235_0.

$\left[\mathrm{FGG}^{+} 10\right]$ A. Favini, G. R. Goldstein, J. A. Goldstein, E. Obrecht, and S. Romanelli. Elliptic operators with general Wentzell boundary conditions, analytic semigroups and the angle concavity theorem. Math. Nachr. 283 (2010), 504-521. https://doi.org/10.1002/mana.200910086.

[FGGR02] A. Favini, G. R. Goldstein, J. A. Goldstein, and S. Romanelli. The heat equation with generalized Wentzell boundary condition. J. Evol. Equ. 2 (2002), 1-19. https://doi.org/10.1007/s00028-002-8077-y.

[Gol06] G. R. Goldstein. Derivation and physical interpretation of general boundary conditions. Adv. Differential Equations 11 (2006), 457-480.

[Gre87] G. Greiner. Perturbing the boundary conditions of a generator. Houston J. Math. 13 (1987), 213229. https://www.math.uh.edu/ hjm/vol13-2.html.

[Nag90] R. Nagel. The spectrum of unbounded operator matrices with nondiagonal domain. J. Funct. Anal. 89 (1990), 291-302. http://dx.doi.org/10.1016/0022-1236(90)90096-4.

[Paz83] A. Pazy. Semigroups of Linear Operators and Applications to Partial Differential Equations, Appl. Math. Sci., vol. 44. Springer (1983). https://doi.org/10.1007/978-1-4612-5561-1.

[Tay96] M. E. Taylor. Partial Differential Equations. II: Qualitative Studies of Linear Equations, Appl. Math. Sci., vol. 116. Springer (1996). http://doi.org/10.1007/978-1-4757-4187-2.

Tim Binz, University of Tübingen, Department of Mathematics, Auf der Morgenstelle 10, D-72076 Tübingen, Germany, tibi@fa.uni-tuebingen.de

Klaus-Jochen Engel, University of L'Aquila, Department of Information Engineering, Computer Science and Mathematics, Via Vetoio, I-67100 L'Aquila - Coppito (AQ), Italy, klaus. engel@univaq.it 